\title{
Experiences and lessons learned from the real-world implementation of an HIV recent infection testing algorithm in three routine service-delivery settings in Kenya and Zimbabwe
}

Mariken M. de Wit ${ }^{1 *}$, Brian Rice ${ }^{1}$, Kathryn Risher ${ }^{1}$, Susie Welty ${ }^{2}$, Wanjiru Waruiru², Sitholubuhle Magutshwa ${ }^{3}$, John Motoku4, Daniel Kwaro ${ }^{5}$, Benard Ochieng ${ }^{5}$, Georges Reniers ${ }^{1}$, Frances Cowann ${ }^{3,6}$, George Rutherford ${ }^{2}$, James R. Hargreaves ${ }^{1}$ and Gary Murphy ${ }^{7}$

\begin{abstract}
Introduction: Testing for recent HIV infection can distinguish recently acquired infection from long-standing infections. Given current interest in the implementation of recent infection testing algorithms (RITA), we report our experiences in implementing a RITA in three pilot studies and highlight important issues to consider when conducting recency testing in routine settings.

Methods: We applied a RITA, incorporating a limited antigen (LAg) avidity assay, in different routine HIV servicedelivery settings in 2018: antenatal care clinics in Siaya County, Kenya, HIV testing and counselling facilities in Nairobi, Kenya, and female sex workers clinics in Zimbabwe. Discussions were conducted with study coordinators, laboratory leads, and facility-based stakeholders to evaluate experiences and lessons learned in relation to implementing recency testing.
\end{abstract}

Results: In Siaya County 10/426 (2.3\%) of women testing HIV positive were classified as recent, compared to 46/530 (8.7\%) of women and men in Nairobi and 33/313 (10.5\%) of female sex workers in Zimbabwe. Across the study setting, we observed differences in acceptance, transport and storage of dried blood spot (DBS) or venous blood samples. For example, the acceptance rate when testing venous blood was $11 \%$ lower than when using DBS. Integrating our study into existing services ensured a quick start of the study and kept the amount of additional resources required low. From a laboratory perspective, the LAg avidity assay was initially difficult to operationalise, but developing a network of laboratories and experts to work together helped to improve this. A challenge that was not overcome was the returning of RITA test results to clients. This was due to delays in laboratory testing, the need for multiple test results to satisfy the RITA, difficulties in aligning clinic visits, and participants opting not to return for test results.

\footnotetext{
* Correspondence: Mariken.dewit@wur.nl

${ }^{1}$ London School of Hygiene and Tropical Medicine, London, UK

Full list of author information is available at the end of the article
}

C C The Author(s). 2021 Open Access This article is licensed under a Creative Commons Attribution 4.0 International License, which permits use, sharing, adaptation, distribution and reproduction in any medium or format, as long as you give appropriate credit to the original author(s) and the source, provide a link to the Creative Commons licence, and indicate if changes were made. The images or other third party material in this article are included in the article's Creative Commons licence, unless indicated otherwise in a credit line to the material. If material is not included in the article's Creative Commons licence and your intended use is not permitted by statutory regulation or exceeds the permitted use, you will need to obtain permission directly from the copyright holder. To view a copy of this licence, visit http://creativecommons.org/licenses/by/4.0/ The Creative Commons Public Domain Dedication waiver (http://creativecommons.org/publicdomain/zero/1.0/) applies to the data made available in this article, unless otherwise stated in a credit line to the data. 
Conclusion: We completed three pilot studies using HIV recency testing based on a RITA in Kenya and Zimbabwe.
The main lessons we learned were related to sample collection and handling, LAg avidity assay performance,
integration into existing services and returning of test results to participants. Our real-world experience could
provide helpful guidance to people currently working on the implementation of HIV recency testing in sub-Saharan
Africa.

Keywords: HIV, Recency testing, RITA, Zimbabwe, Kenya, Implementation

\section{Introduction}

To identify populations and areas where HIV transmission is occurring, it is helpful to distinguish among newly diagnosed persons those who have acquired infection recently from those with a longer-standing infection. Several HIV recency tests have been developed to make such a distinction, including the HIV limiting antigen (LAg) avidity assay [1, 2]. Using LAg avidity assay test results alone (i.e. in the absence of viral load and antiretroviral therapy (ART) testing) to determine recency status is associated with an unacceptably high proportion false recent results (also known as false recency rate), which can lead to overestimation of HIV incidence without appropriate adjustment [3]. Recent infection testing algorithms (RITA) combine multiple test results to improve the classification of recent infections and decrease the proportion false recent. Since 2015, the United Nations Joint Programme on HIV/AIDS (UNAIDS) and the World Health Organization (WHO) have recommended the inclusion of viral load testing in RITAs to estimate incidence [4]. A study conducted in Kenya and South Africa showed that inclusion of ART exposure status in the RITA may further improve incidence estimates [5]. While these additional steps improve the accuracy of incidence estimates, they also make implementation of the RITA more challenging.

RITAs have been used in several settings, for example in trials [6], routine surveillance $[7,8]$ and in population surveys in sub-Saharan Africa [9-12]. In 2019, the U.S. President's Emergency Plan for AIDS Relief (PEPFAR) called for recency testing to be implemented among all newly diagnosed individuals across all sites in supported countries that had achieved certain basic progress goals [13]. In some countries, recency test results are returned to participants, based on either the LAg avidity assay or the full RITA [14]. Previous studies have outlined some of the issues that need to be considered when conducting recency testing and when interpreting test results. These issues include differences in recency classification by sample type [15], the effect of storage temperature on dried blood spot (DBS) samples [16], and the need to include multiple tests in a RITA [3]. However, to the best of our knowledge, there are no published studies about real-word experiences in implementing recency testing in routine service-delivery settings.
We conducted three independent, but linked, pilots of HIV recency testing in a variety of routine serviceprovision contexts in Kenya and Zimbabwe in 2018. The three study settings were antenatal clinics providing prevention of mother-to-child HIV transmission services in Siaya County, Kenya, in HIV testing and counselling clinics in Nairobi, Kenya, and in a national programme for female sex workers in Zimbabwe. Here, we discuss our experiences with the implementation of a RITA and share lessons learned to inform other initiatives aiming to integrate recency testing into their routine programme services.

\section{Methods \\ Study population}

The three pilot studies were conducted in different routine service-provision settings (Table 1). In Siaya County, Kenya we tested pregnant women aged 13 years or older who tested HIV positive at their antenatal care visit at one of 14 antenatal care facilities in Gem subcounty. To determine HIV recency status, the Maxim HIV-1 LAgAvidity enzyme-linked immunoassay (EIA) for venous blood was used. Recency test results were not returned to the participants. This pilot was conducted in collaboration with the Kenya Medical Research Institute (KEMR I), and participants were linked to the KEMRI/Centers for Disease Control and Prevention (CDC) Siaya Health and Demographic Surveillance Site (Siaya HDSS).

In Nairobi, Kenya, we tested clients who tested HIV positive at any of 14 Eastern Deanery AIDS Relief Programme (EDARP) HIV testing and counselling facilities. EDARP has been providing community-based HIV and tuberculosis prevention, testing, treatment and care services in eastern Nairobi for the past 24 years [18]. Testing was done using Maxim HIV-1 LAg-Avidity EIA assay on dried blood spots (DBS). Results were returned to the client at their next clinic visit. Detailed methods and results from this pilot have been described elsewhere [19].

In Zimbabwe, our study focused on female sex workers testing HIV positive at any of six static sites of the Sisters with a Voice programme. Both venous blood samples and capillary DBS samples were taken. When a woman preferred not to give a venous blood sample, only a DBS was taken. HIV recency status was 
Table 1 Three pilots of HIV recent infection testing in routine service settings (adapted from [17]

\begin{tabular}{|c|c|c|}
\hline Siaya County, Kenya & Nairobi, Kenya & Sisters with a Voice, Zimbabwe \\
\hline Study population & Study population & Study population \\
\hline $\begin{array}{l}\text { Pregnant women seeking antenatal care in } 14 \\
\text { medical facilities }\end{array}$ & $\begin{array}{l}\text { Clients attending any of the } 14 \text { EDARP HTC } \\
\text { facilities }\end{array}$ & $\begin{array}{l}\text { FSW attending one of six static facilities of } \\
\text { the Sisters with a Voice Programme }\end{array}$ \\
\hline Collaborative partner & Collaborative partner & Collaborative partner \\
\hline $\begin{array}{l}\text { Kenya Medical Research Institute (KEMRI) and the } \\
\text { KEMRI/CDC Siaya HDSS }\end{array}$ & Eastern Deanery AIDS Relief Programme (EDARP) & $\begin{array}{l}\text { Centre for Sexual Health and HIV AIDS } \\
\text { Research Zimbabwe (CeSHHAR-Zimbabwe) }\end{array}$ \\
\hline Study period & Study period & Study period \\
\hline February - November 2018 & March - November 2018 & June - November 2018 \\
\hline Assay & Assay & Assay \\
\hline Maxim HIV-1 LAg-Avidity EIA venous blood & Maxim HIV-1 LAg-Avidity EIA DBS & $\begin{array}{l}\text { Maxim HIV-1 LAg-Avidity EIA venous blood } \\
\text { \& Maxim HIV-1 LAg-Avidity EIA DBS }\end{array}$ \\
\hline Inclusion criteria & Inclusion criteria & Inclusion criteria \\
\hline \multirow[t]{3}{*}{$\begin{array}{l}\text {-Women aged } 13 \text { or older seeking antenatal care } \\
\text { in one of the included medical facilities in Siaya } \\
\text { County } \\
\text { - Willing and able to provide informed consent } \\
\text { - Received an HIV-antibody positive test result }\end{array}$} & $\begin{array}{l}\text { - Aged } 18 \text { or older } \\
\text { - Unknown HIV status prior to visit } \\
\text { - Attending an EDARP HTC facility } \\
\text { - Willing and able to provide informed consent } \\
\text { - Received an HIV-antibody positive test result, or } \\
\text { presumptive positive }\end{array}$ & $\begin{array}{l}\text { - FSW aged } 18 \text { or older } \\
\text { - Willing and able to provide informed } \\
\text { consent } \\
\text { - Received an HIV-antibody positive test } \\
\text { result }\end{array}$ \\
\hline & Exclusion criteria & Exclusion criteria \\
\hline & $\begin{array}{l}\text { - Indeterminate HIV result } \\
\text { - Not willing to enrol on follow-up at facility } \\
\text { - Taking pre-exposure prophylaxis }\end{array}$ & $\begin{array}{l}\text { - Indeterminate HIV result } \\
\text { - Prior history of testing HIV-positive (>1 } \\
\text { year ago) } \\
\text { - On ART }\end{array}$ \\
\hline Specimen collection and testing & Specimen collection and testing & Specimen collection and testing \\
\hline $\begin{array}{l}\text { - Facility nurse or laboratory phlebotomist drew a } \\
\text { maximum of } 10 \mathrm{ml} \text { of venous blood } \\
\text { - Samples packed and transferred to KEMRI-Centre } \\
\text { for Global Health Research HIV Research Labora- } \\
\text { tory in Kisumu on a daily basis where they were } \\
\text { processed and stored for testing }\end{array}$ & $\begin{array}{l}\text { Study nurse drew } 6 \mathrm{~mL} \text { of venous blood collected } \\
\text { in an ethylenediaminetetraacetic (EDTA) tube and } \\
\text { a pipette was used to dispense venous blood on } \\
\text { two Whatman }{ }^{\text {TM }} 903 \text { Snap-Apart Cards with } 5 \text { dried } \\
\text { blood spots (DBS) of } 70 \mu \mathrm{LL} \text { each, for a total of } 10 \\
\text { filled spots per participant }\end{array}$ & $\begin{array}{l}\text { - Study or clinic nurse drew venous blood } \\
\text { collected in an ethylenediaminetetraacetic } \\
\text { (EDTA) tube. } \\
\text { - DBS samples were collected from } \\
\text { fingerprick on one Whatman }{ }^{\mathrm{TM}} 903 \text { Snap- } \\
\text { Apart Cards with } 5 \text { dried blood spots (DBS) } \\
\text { of } 70 \mathrm{\mu L} \text {. } \\
\text { - Both samples were packed and transferred } \\
\text { to laboratory in Harare within } 36 \mathrm{~h} \text { and } \\
\text { stored at -20C or below prior to testing }\end{array}$ \\
\hline
\end{tabular}

determined using Maxim HIV-1 LAg-Avidity EIA assays for venous blood as well as for DBS as both samples were taken. We compared acceptance rates between DBS and venous blood using McNemar's test. Results of venous blood tests were to be made available at Sisters clinics within 2 weeks for timely return to participants. This pilot was conducted in collaboration with the Centre for Sexual Health and HIV/AIDS Research (CeSHHAR). CeSHHAR, on behalf of the Zimbabwean National AIDS Council and Ministry of Health and Child Care, runs the national Sisters with a Voice programme for female sex workers, providing a range of HIV-related services [20]. The three studies were linked through shared protocol design, and through joint coordination via a cross-pilot working group. The working group held bi-weekly conference calls which provided a platform for shared learnings and raising and responding to questions and issues as they arose.

\section{Recent infection testing algorithm}

Figure 1 presents the RITA we integrated into routine service. All individuals who were tested HIV positive were assessed for eligibility. In Nairobi and Zimbabwe, we did not recruit individuals for assessment of recent infection if they self-reported a prior positive HIV antibody test (in Zimbabwe this had to be $>1$ year ago) or if they reported taking ART. We classified these individuals as having a non-recent infection. We conducted LAg avidity testing on the remaining participants and classified samples with a normalized optical density (ODn) > 1.5 as long-standing infections. For samples with an $\mathrm{ODn} \leq 1.5$, we conducted viral load testing and classified those with a viral load $<1000$ as long-standing infections and those with a viral load $\geq 1000$ copies $/ \mathrm{mL}$ as potentially positive for recent infection. We further characterised potentially positive samples by ART status determined by testing for the presence of ART 


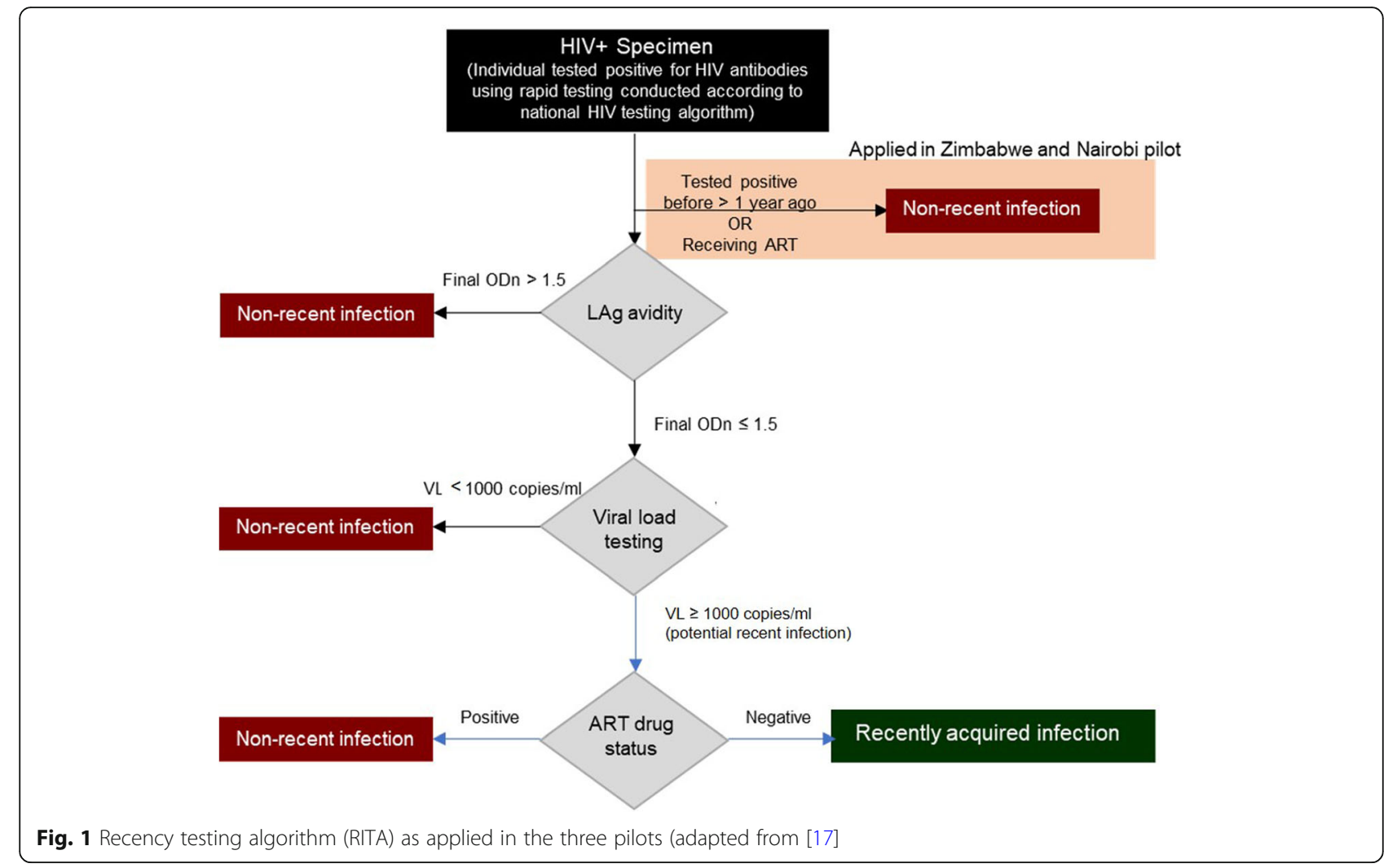

metabolites (Nairobi), checking the clinic record (Siaya County), or self-report (Zimbabwe) and classified samples from people on ART as long-standing infections and those from people not on ART as positive for recent infection.

\section{LAg avidity assay}

Recency assays are available for venous blood and DBS samples [21-24]. Sample collection via DBS is regarded as a simpler and more efficient method than venous blood sampling, because blood drops are easier to collect and DBS cards can be transported and stored dry at room temperature. Venous blood results are seen as more reliable (as they are less sensitive to haematocrit levels than DBS) but require venepuncture, processing to separate plasma and serum from whole blood, and maintenance of a cold chain during transportation and storage.

Both assay types were used in this study (Table 1). The Maxim HIV-1 LAg-Avidity EIA (Maxim Biomedical, Inc., Rockville, Maryland, USA) assays were performed according to the manufacturer's instructions for use [25]. Wells of a microtiter plate were coated with a limited amount of multi-subtype recombinant HIV-1 antigen to which antibodies bind. Specimens were diluted and incubated for $60 \mathrm{~min}$ before a disassociation buffer was added for $15 \mathrm{~min}$ to remove weakly-bound antibodies. A goat anti-human, horseradish peroxidase (HRP)-conjugated IgG was then added, which bound to any remaining antibodies. A colour, proportionate to the amount of HRP, was generated by adding a tetramethylbenzidine substrate. Optical densities were measured for each sample and normalised by use of a calibrator specimen. The median was calculated from triplicate testing of the calibrator on each plate. The normalized optical density (ODn) was calculated by dividing the OD for each specimen by the median calibrator OD. Specimens with an ODn value $\leq 2.0$ were retested in triplicate from a fresh dilution, as required by the testing procedures. For these specimens the final ODn value was the median value of the triplicate test results. Anti-HIV serology was performed on specimens showing ODn value $<0.4$ to confirm HIV antibody positivity.

\section{Viral load and ART metabolite testing}

Viral load was measured using the laboratory-based Abbott m2000 RealTime system (Abbott Laboratories, Abbott Park, Illinois, USA), Roche Cobas AmpliPrep/Cobas Taqman (Roche Diagnostics International AG, Basel, Switzerland), or similar automated platforms, according to manufacturers' instructions.

As including information on exposure to ART could improve RITA performance through lowering falserecent misclassification, Nairobi study plasma samples 
assessed as potentially recent positive were tested for the presence of antiretroviral metabolites at the Division of Clinical Pharmacology at the University of Cape Town. Antiretroviral metabolites, including lopinavir, ritonavir, nevirapine, efavirenz, indinavir, saquinavir, zidovudine, lamivudine, and stavudine, were quantified by a robust simultaneous liquid chromatography/tandem mass spectrometry method [26].

\section{Evaluation}

To monitor the laboratory outputs and ensure testing practices were of good quality, a LAg avidity assay expert (Dr Gary Murphy, expert consultant) reviewed the outputs for a random sample of the plates. This included evaluating the control samples against the permitted criteria, the reproducibility of results, the comparison of initial versus confirmatory results, and the correct entering of results. One of the authors (MdW) met with the study coordinators of the Zimbabwe and Nairobi pilot and observed study enrolment in a clinic as well as the laboratory procedures in Zimbabwe. Towards the end of the study, informal one-to-one discussions were conducted with study coordinators, laboratory leads, and facility-based stakeholders to construct an image of people's experiences with the different aspects of the project. Discussion topics included experiences with communication, field work, laboratory work, and coordination, as well as recommendations and lessons learned. Together, this provided a picture of how the study was conducted in actuality and how it could be improved in the future. A more detailed description of this study has been provided elsewhere [17].

\section{Results}

Table 2 provides a summary of study recruitment, test results, and return of these results. Across 34 pilot sites, in total 1269 people testing HIV positive completed the RITA, of whom 89 (7.0\%) were classified as positive for recent infection. A detailed description of the test results (quantitative focus) have been presented elsewhere [17].

\section{Sample types and transportation}

Clear differences were observed in the collection of venous blood and DBS. In our pilot study in Zimbabwe, where both sample types were collected from participants, acceptance rates for recency testing were lower when venous blood collection was required for a sample ( $n=313,61 \%$ ) compared to a finger prick for a DBS sample $(n=367,72 \%)(p<0.001)$. After sample collection, venous blood samples needed to be rapidly transported to the laboratory so that they could be centrifuged and stored in a freezer below $-20{ }^{\circ} \mathrm{C}$ to avoid haemolysis. This proved to be challenging given recruitment sites were often hours from the laboratory and required careful planning. The venous blood and DBS samples were collected and transported at the same time.

In Siaya county, sites were often in rural locations, and venous blood samples were transported by motorcycle in a cooler box in a backpack to avoid damage caused by uneven roads. Transport was daily, as samples had to get to the laboratory within $5 \mathrm{~h}$ of collection, and took 1 to $2 \mathrm{~h}$. Measures to reduce transportation time, such as having drivers stationed at a central location and available to all sites, were shown to work as none of the collected samples were rejected by the laboratory for poor quality.

In Nairobi, where sites were closer in proximity, a driver collected samples from all sites for delivery to the laboratory in the morning. If any additional samples were taken later during the day, a courier picked them up from the facilities.

Table 2 Recruitment, test results, and return of test results by pilot study

\begin{tabular}{llll}
\hline & Siaya County & Nairobi & Zimbabwe $^{\mathbf{a}}$ \\
\hline Participating sites & 14 & 14 & 6 \\
Eligible for HIV test & 2365 & 50,561 & 4349 \\
Tested HIV positive $^{\text {b }}$ & 445 & 628 & 510 \\
Completed RITA $_{\text {Recent infection positive prior to ART verification }}$ & 426 & 530 & 313 \\
Number of recent infections after ART verification & & $48(9.1 \%)$ & $33(10.5 \%)$ \\
Completed RITA results returned to client & $11(2.6 \%)$ & $46(8.7 \%)$ & $33(10.5 \%)$ \\
$\quad$ Recent infection & $10(2.3 \%)$ & & \\
$\quad$ Long-standing infection & & $29(60.4 \%)$ & $9(27.3 \%)$ \\
\hline
\end{tabular}

NA not applicable

${ }^{a}$ Results from venous blood tests

b Includes people testing HIV positive for the first time in Nairobi and Zimbabwe

CART status was externally verified in Siaya County and Nairobi but not Zimbabwe 


\section{Laboratory performance}

We trained staff to perform the LAg avidity assays correctly. Despite this training, and despite most laboratory staff being familiar with similar assays, laboratory staff in all three settings stated that performing the LAg avidity assay correctly was difficult and required a lot of precision. In one of the pilot studies, we repeatedly encountered invalid results from the LAg avidity assay and had to temporarily pause recruitment during month four of the study. To keep delays to a minimum, we transferred testing to a back-up laboratory during the temporary pause. By comparing operational procedures between the original and back-up laboratories, we were able to identify causes for the failed runs. The main reason for invalid results was found to be incorrect calibration and maintenance of the microplate washer. The washer was replaced as a result of this investigation and testing could be resumed. We learned that ensuring that these items of equipment are available, well maintained, properly calibrated and used correctly are critical steps before testing starts.

In recognition that a number of challenges across the pilots were arising in relation to collecting, testing, and returning samples, we introduced a regular call that brought together the three in-country pilot teams, as well as the study team based in London. Prior to this, we were running the pilots as linked but independent studies. The laboratory technicians appreciated having such a network of expertise as they were able to discuss the problems they encountered, share advice, and gain support.

Another lesson we identified retrospectively was the potential utility of participating in an external quality assurance scheme. A scheme for the performance of Maxim and Sedia LAg assays is run by Duke University's External Quality Assurance Program Oversight Laboratory (EQAPOL) [27]. This scheme distributes specimens of known reactivity to participants who then return the results of their testing to EQAPOL. These results are analysed to determine agreement with the expected results thereby helping identify whether laboratories are performing tests correctly. Signing up to this early on in our study might have prevented the recruitment pause.

In Table 3, we summarise issues that can occur when performing recency testing in laboratories. We present a combination of challenges we encountered in this study and challenges encountered by our team's LAg avidity assay expert in similar studies.

\section{Organisation of fieldwork}

Recency testing is increasingly implemented as part of existing programmes and/or services [13]. As described in the methods, we integrated recency testing into antenatal care services, a general HIV testing service, and a sex workers programme. Embedding recency testing in existing structures made it possible to get the study started quickly and also helped to calculate the expected sample size given that the number of clients visiting the programme was known from previous experience. In the Nairobi and Zimbabwe pilots, observed recruitment was similar to expected (as calculated in our original protocols based on programme activity) recruitment (600 expected and 628 recruited in Nairobi; 600 expected and 510 recruited in Zimbabwe). In Siaya County, recruitment was lower than anticipated (864 expected and 445 recruited). The differences between our observed and expected recruitment numbers reflect how the pilot studies were reliant upon existing programme activity. For example, in Zimbabwe, a delay in funding for programme activities resulted in our study commencing later than expected.

Embedding our pilots within established programmes also required, at times, client flows to be adapted and additional staff to be accommodated. The study required the inclusion of additional questions to the routine client question list. The inclusion of recency testing-related questions in some settings lengthened the routine consulting period and may have resulted in increased hesitation by clients to participate in the study. To overcome potential client concerns, staff were trained to help people find their way around the facility and to answer questions as they arose. Additional study requirements in some settings resulted in an extra data clerk having to be accommodated, which in turn resulted in having to adapt clinic-space organisation. The impact of new tests and personnel on clinic organisation can negatively impact client flow [28].

Study recruitment was observed to vary across facilities and time. In Siaya County, routine checks were conducted to see whether we were reaching everybody attending the facilities. This was done by comparing the number of participants enrolled in the study to the number of participants recorded in the antenatal care register. If a potential participant were missed, she was reached during the subsequent visit. In Nairobi, reminders for recruitment were implemented into the electronic medical records system to ensure nobody was missed. In hindsight, it would have been useful to have trained study staff to conduct real-time data monitoring to pick up on variations in recruitment and data entry errors quickly. By doing this, low recruitment or acceptance rates could be identified earlier on, and resolved.

\section{Return of test results}

The timely return of test results to facilities and clients proved to be particularly challenging. We had planned in Nairobi and Zimbabwe to schedule the return of the recency test result to clients during their next clinic 
Table 3 Potential issues when implementing a RITA, with recommendations and additional considerations

\begin{tabular}{ll}
\hline $\begin{array}{l}\text { Issue } \\
\text { assay }\end{array}$ & Assay availability may be limited \\
& $\begin{array}{l}\text { Assay kit may not be able to be } \\
\text { imported into country }\end{array}$ \\
& Assay transport within country \\
$\begin{array}{l}\text { Sample } \\
\text { types }\end{array}$ & Specimen transport within country
\end{tabular}

Training and Before testing is undertaken staff performance should be trained in the performance of the assay

Assay only requires relatively basic laboratory equipment however it is very sensitive to issues such as inadequate washing.

Additional items such as bloodletting equipment and Dried Blood spot cards are required

Reagents should be stored as per assays instructions for use (IFU).

In use reagents

Testing must be performed in accordance with the assays 'instruction for use'

\section{Quality Temperature control}

control

Internal quality control

Analysis of quality control data

\section{Recommendation}

Discuss with assay manufacturer well in advance of launch of study the number of assays required.

Ensure import permits are in place at an early stage.

Ensure cold chain is maintained for the assay when it is transported to local labs.

Specimens should be transported under cold chain. Serum/plasma specimens for LAg testing should be separated locally before transport. DBS specimens should ensure that the specimen is fully dried before transport and maintained with a desiccant.

Although a standard ElA this assay has multiple pass/fail criteria and troubleshooting errors can be complex. All users should receive training from an experienced user before undertaking 'real-world' testing. CDC offer training panels to help users achieve competency in the assay and help should be sought from CDC.

Ensure all required equipment is available and is fully serviced, maintained and calibrated. Equipment to be used should be itemised before testing begins and reviewed by an experienced individual to ensure it is suitable for use.

These are often outside of the control of the laboratory, but it should be ensured that the equipment used is of the right type, within expiry date and stored appropriately.

Reagents are stored at a variety of temperatures and these must be adhered to. Temperature monitoring should be in place for all freezers and refrigerators.

Wash reagents must be made as per the assays 'IFU'. Unless the whole kit is being used at one time then unused reagents must be returned to appropriate storage conditions as soon as possible.

LAg assay is a generic term and at least two manufacturers supply a version of the assay. It is critical that the correct method is used for each assay.

LAg assays are very prescriptive on incubation times and temperatures. Assays should be performed using incubators with precise and recorded temperatures rather than incubated in the open lab regardless of the current temperature of the lab.

Although the assays come with control material, users should follow good laboratory practice and include some specimens of known reactivity in every test to ensure reproducibility over time.

The manufacturer supplied analysis software

\section{Additional considerations}

This will require an understanding of the population to be studied and the anticipated confirmation rate.

Delays to imports may affect performance of assay if it is not stored in appropriate condition when awaiting customs clearance.

Minimising number of laboratories performing the assay may make this easier.

Depending upon other tests required, different specimens may need to be transported under different conditions.

This training will not only help support the testing procedure but also the analysis tools of the associated software. Individuals should be monitored regularly to confirm compliance with the testing protocol. Testing laboratories must ensure that Standard operating procedures are in place for the assay detailing all steps and conditions undertaken in their lab to allow troubleshooting of issues and enhance data analysis.

Service contracts may not be in place for some pieces of equipment so monitoring the performance of equipment is critical to ensure it is performing as expected. When considering equipment even 'common' items such as pipettes should be serviced and calibrated before use.

Details of all ancillary equipment should be recorded and available for inspection.

Ensure that supplies such as water are appropriate for use following assay's 'IFU'.

As well as two manufacturers there are different versions of the kit dependent on sample type being used plasma/serum or DBS. The appropriate method must be followed for these.

Regular monitoring of equipment temperatures should be recorded and incubators confirmed to have reached temperature before starting assay.

These results should be plotted and analysed to look for any trends in performance. 
Table 3 Potential issues when implementing a RITA, with recommendations and additional considerations (Continued)

\begin{tabular}{|c|c|}
\hline Issue & Recommendation \\
\hline & $\begin{array}{l}\text { will provide quality control data on the assay } \\
\text { performance. Users should ensure that they } \\
\text { enter data correctly and ensure upper } \\
\text { reading OD of the spectrophotometer is } \\
\text { accurately entered. Users should review data } \\
\text { on their own internal quality control } \\
\text { independently as this is not captured } \\
\text { longitudinally by company software. }\end{array}$ \\
\hline External Quality Assurance & $\begin{array}{l}\text { Each testing laboratory should partake in an } \\
\text { external quality assurance programme for the } \\
\text { assay. This will help provide confidence in the } \\
\text { results issued by each laboratory. The } \\
\text { programme for this assay is available via } \\
\text { EQAPOL. }\end{array}$ \\
\hline
\end{tabular}

Assay failures

\section{Reporting Confounding factors results}

Assay linked analysis software

Confirmatory testing

Unusual results

Laboratories should record and share any failed runs with assay specialists. This will help to identify if there is any systematic error occurring with the assay and enhance the ability to troubleshoot the assay.

Users should ensure they are using the correct analysis software associated with the assays. Each assay has different validation criteria and use of the incorrect manufacturer's software may lead to errors in assay interpretation and validation.

Confirmatory testing must be performed from a freshly diluted specimen. It must not be performed from the dilution prepared for the screening assay.

All unusual results should be investigated, and retesting undertaken if warranted. Unusual results may include specimens which offer a low OD (which require retesting to confirm the specimen contains antibodies to HIV-1) or samples where the screening ODn is significantly different from that of the confirmatory test. Investigations should be undertaken to confirm that the correct specimen was retested (or tested initially).

Users should be aware that a number of factors can affect the performance of the test and these should be considered when analysing data. These include factors such as anti-retroviral treatment of individuals and HIV subtype prevalence.

Assay specific performance data

Over-interpretation of the data

\section{Additional considerations}

Users should ensure that details of all equipment used (Including pipettes etc) is recorded and the items are identifiable by serial number. Batch number of assay should be recorded (this will be performed by the company supplied software if used).

Users should also be aware to use the correct software for plasma/serum specimens and for DBS specimens as these differ.

Reviewing confirmatory test data can help determine how well an assay is being performed as the replicates should be very close. A wide range of values on the confirmatory test may indicate poor pipetting competency of the tester or poor reliability of the pipettes.

Many assays will not differentiate HIV-1 from HIV-2 so care should be taken where HIV-2 is prevalent.
Users should be very clear as to which assay was used and the cut-offs applied to their data as this impacts on the period in which recent HIV infection can be inferred by the assay. This is also important where multiple HIV-1 clades are prevalent.

Users should be aware of the limitations of the assay and not over interpret the data. For example, a very low ODn may indicate a very recent $\mathrm{HIV}$ infection or that the individual is not HIV infected. As per the algorithm these specimens should be submitted for HIV diagnostic confirmatory testing.
Results should not be issued without being part of an algorithm to reduce the potential for misclassification.

Application of data trends should take into account potential confounders such as changes in the population, the application of different HIC screening tests over time and the use of different LAg assays in previous surveys. 
visits. However, we found that this was not always feasible, and clients often did not return for their results. In Nairobi and Zimbabwe, respectively, 402 (76\%) and 33 (11\%) of results were finally returned to the client. In Nairobi, only 7 (1.7\%) of results were returned within 1 month and 266 (66.2\%) of results were returned more than 3 months after recruitment of the participant. In Zimbabwe, return time was highly variable and mostly dependent on when women returned to the clinic. Women frequently did not return to the clinic for results and gave false contact details which hampered followup. The late return of a test result may have a negative impact on the value of the result to the client and their utility in informing prevention activities such as partner notification [19].

Delays in laboratory testing occurred due to failed assay runs or increased waiting time for the samples to be tested when recruitment was slow. As recency testing kits are expensive, we aimed to minimise the amount of reagents used. With every assay using 11 control wells, it proved efficient to run plates only when enough samples were available to fill the plate. During periods when recruitment was slow, this resulted in delays. This not only delayed return of test results but also meant that samples were stored for different durations, potentially affecting results if storage conditions were not optimal.

Another significant challenge to the timely return of test results was the need for multiple assays to complete the RITA. Viral load tests could be run in our participating laboratories, but ART metabolite testing required samples to be sent to the University of Cape Town in South Africa. This proved to be difficult for two reasons: obtaining import and export permits within the study period, and a testing backlog at the University of Cape Town. Although we planned to conduct ART metabolite testing across all three pilots, due to these two challenges, it ended up only being carried out for the Nairobi pilot. Having an in-country facility that can conduct ART metabolite testing would have helped overcome these issues, although this might not be worth the costs unless it were part of a holistic infrastructure development. Apart from additional administrative and logistical work, shipment for ART metabolite testing also required high standards of storage. This raises the question of what the optimal balance is between completing the full RITA and quickly returning test results to participants. An important consideration for a RITA result that lacks an ART test component is the information and counselling provided upon the return of recency test result to a client. The interpretation of a test result is complicated regardless of the results because of the long window of recent infection and the risk of false recent results. This becomes even more complicated when exposure to ART in unknown and has to be kept in mind when giving feedback to clients.

\section{Discussion}

We conducted three pilot studies of HIV recency testing in routine service-provision settings in Kenya and Zimbabwe. A total of 1269 people completed the RITA across the three studies, of whom $7 \%$ were found to have a recent infection. The main implementation challenges we faced related to sample collection and handling, LAg avidity assay performance, integration into existing services, and returning of test results to clients. In this paper, we provide a detailed analysis of challenges related to laboratory procedures, together with recommendations on how to address, and preferably avoid, these.

The choice to use DBS or venous blood samples affected acceptance rates, transportation, and storage. Although the acceptance rate for venous blood was lower than for DBS in Zimbabwe, challenges relating to the rapid transportation and storage of venous blood samples were overcome through careful planning and coordination. Ensuring the correct performance of the LAg avidity assay and obtaining high quality results were more difficult than expected, underscoring the need for adequate training and competency assessment of staff members performing the assay. When problems with equipment came to light, having a network of laboratories and experts working together was useful to troubleshoot problems, promote collaboration and provide back-up services. Because the pilots were integrated into existing services, the study could start relatively quickly. However, we also came across the downside of being dependent on other programmes and having to adapt routine practices. Returning RITA test results to participants in a timely manner proved to be particularly challenging due to delays in laboratory testing, the need for multiple test results, alignment with clinic visits, and participants not returning for test results. As late return of test results may lessen the value of the results for clients, and for informing activities such as partner notification, future programmes planning to return test results should take account of these issues in their planning stage. Return of the test result had no impact on treatment, as all participants that tested HIV positive were initiated on an ART regimen, regardless of their recency status.

Our study was one of the first to implement recency testing in routine settings in sub-Saharan Africa. As recency testing is currently being scaled up in PEPFARsupported countries to include all people with newly diagnosed HIV infection [13], our real-world experience and lessons learned can provide helpful guidance to the implementation of HIV recency testing. 


\section{Conclusions}

Our analysis showed that recency testing can be successfully implemented in routine settings. However, a number of challenges can be expected: challenges that can be overcome through careful preparation, continuous monitoring of test results to ensure high quality, good communication, intensive coordination with routine service providers, and quick turn-around of laboratory tests.

\section{Abbreviations}

ART: Antiretroviral therapy; CDC: Centers for Disease Control and Prevention; CeSHHAR: Centre for Sexual Health and HIV/AIDS Research; DBS: Dried blood spot; EDARP: Eastern Deanery AIDS Relief Programme; EIA: Enzyme-linked immunoassay; EQAPOL: External Quality Assurance Program Oversight Laboratory; HDSS: Health and Demographic Surveillance Site; HRP: Horseradish peroxidase; IFU: Instructions for use; KEMRI: Kenya Medical Research Institute; LAg: Limiting antigen; ODn: Normalized optical density; PEPFAR: U.S. President's Emergency Plan for AIDS Relief; RITA: Recent infection testing algorithm; WHO: World Health Organisation

\section{Acknowledgements}

We gratefully acknowledge all the members of the MeSH Working Group on routine HIV recent infection testing for their contributions to running these three pilots of recency testing. We also wish to thank the many individuals who participated in our pilots.

\section{Authors' contributions}

MdW wrote the initial draft of the paper. GM supported the laboratory analyses and provided significant input into the paper. BR and $\mathrm{JH}$ conceived, organised, and led the study. MdW, SW, and KR analysed the data. WW, SM, JM, DK and BO coordinated the study. FC, GReniers and GRutherford implemented the work. All authors have read and approved the final manuscript.

\section{Funding}

This study was funded by the Bill and Melinda Gates Foundation (Grant Number: BMGF OPP1120138).

\section{Availability of data and materials}

The datasets generated and/or analysed during the current study are not publicly available due their sensitive nature but are available from the corresponding author on reasonable request

\section{Declarations}

\section{Ethics approval and consent to participate}

Ethical approval was provided locally by the ethical committee of Medical Research Council of Zimbabwe for the Zimbabwe pilot, by Kenyatta National Hospital-University of Nairobi Ethical Review Board for the pilot in Nairobi, and by Kenya Medical Research Institute (KEMRI) Scientific Ethics Review Unit (SERU application 3589) for the pilot in Siaya County. Ethical approval was also obtained at the London School of Hygiene and Tropical Medicine for the Zimbabwe pilot (reference number 14542), Nairobi pilot (reference number 14585) and Siaya Country pilot (reference number 14458) and at the University of California, San Francisco for the Nairobi pilot. All protocols were carried out in accordance with relevant guidelines and regulations. Informed consent was obtained from all participants.

\section{Consent for publication}

Not applicable

\section{Competing interests}

The authors declare that they have no competing interests.

\section{Author details}

${ }^{1}$ London School of Hygiene and Tropical Medicine, London, UK. ${ }^{2}$ University of California, San Francisco, USA. ${ }^{3}$ Centre for Sexual Health and HIV/AIDS Research, Harare, Zimbabwe. ${ }^{4}$ Eastern Deanery AIDS Relief Programme, Nairobi, Kenya. ${ }^{5}$ Kenya Medical Research Institute, Kisumu, Kenya. ${ }^{6}$ Liverpool
School of Tropical Medicine, Liverpool, UK. ${ }^{7}$ Independent consultant in HIV laboratory diagnostics, London, UK.

Received: 6 February 2021 Accepted: 4 June 2021

Published online: 22 June 2021

\section{References}

1. Duong YT, Qiu M, De AK, Jackson K, Dobbs T, Kim AA, et al. Detection of recent HIV-1 infection using a new limiting-antigen avidity assay: potential for HIV-1 incidence estimates and avidity maturation studies. PLoS One. 2012;7(3) [cited 2020 Sep 27]. Available from: https://pubmed.ncbi.nlm.nih. gov/22479384/.

2. Wei X, Liu X, Dobbs T, Kuehl D, Nkengasong JN, Hu DJ, et al. Development of two avidity-based assays to detect recent HIV type 1 seroconversion using a multisubtype gp41 recombinant protein. AIDS Res Hum Retrovir. 2010;26(1):61-71 [cited 2020 Sep 27]. Available from: https://pubmed.ncbi. nlm.nih.gov/20063992/.

3. Kassanjee R, Pilcher CD, Busch MP, Murphy G, Facente SN, Keating SM, et al. Viral load criteria and threshold optimization to improve HIV incidence assay characteristics. AIDS. 2016;30(15):2361-71 [cited 2020 Sep 27]. Available from: https://pubmed.ncbi.nlm.nih.gov/27454561/.

4. UNAIDSNHO. Technical update on HIV incidence assays for surveillance and monitoring purposes. 2015.

5. Kim AA, Rehle T. Short communication: Assessing estimates of HIV incidence with a recent infection testing algorithm that includes viral load testing and exposure to antiretroviral therapy. AIDS Res Hum Retrovir. 2018; 34(10):863-6 [cited 2020 Sep 27]. Available from: https://pubmed.ncbi.nlm. nih.gov/29926735/.

6. Laeyendecker O, Kulich M, Donnell D, Komárek A, Omelka M, Mullis CE, et al. Development of methods for cross-sectional HIV incidence estimation in a large, community randomized trial. PLoS One. 2013;8(11) [cited 2020 Sep 27]. Available from: https://pubmed.ncbi.nlm.nih.gov/24236054/.

7. Hofmann A, Hauser A, Zimmermann R, Santos-Hövener C, BätzingFeigenbaum J, Wildner S, et al. Surveillance of recent HIV infections among newly diagnosed HIV cases in Germany between 2008 and 2014. BMC Infect Dis. 2017;17(1) [cited 2020 Sep 27]. Available from: https://pubmed. ncbi.nlm.nih.gov/28693564/.

8. Aghaizu A, Murphy G, Tosswill J, DeAngelis D, Charlett A, Gill ON, et al. Recent infection testing algorithm (RITA) applied to new HIV diagnoses in England, Wales and Northern Ireland, 2009 to 2011. Eurosurveillance. 2014; 19(2) [cited 2020 Sep 27]. Available from: https://pubmed.ncbi.nlm.nih. gov/24457006/.

9. Kimanga DO, Ogola S, Umuro M, Ng'ang'a A, Kimondo L, Murithi P, et al. Prevalence and incidence of HIV infection, trends, and risk factors among persons aged 15-64 years in Kenya: Results from a nationally representative study. J Acquir Immune Defic Syndr. 2014;66(SUPPL. 1) [cited 2020 Sep 27 ]. Available from: https://pubmed.ncbi.nlm.nih.gov/24445338/.

10. Shishana O, Rehle T, Simbay L, Zuma K, Jooste S, Zungu N, et al. South African national HIV prevalence, incidence and behaviour survey, 2012. 2014.

11. Centers for Disease Control and Prevention (CDC), ICAP, Columbia University Mailman School of Public Health, (Swaziland) M of H. Swaziland HIV Incidence Measurement Survey (SHIMS): First Findings Report, November 2012. 2012.

12. Grebe E, Welte A, Johnson LF, Van Cutsem G, Puren A, Ellman T, et al. Population-level HIV incidence estimates using a combination of synthetic cohort and recency biomarker approaches in KwaZulu-Natal, South Africa. PLoS One. 2018;13(9) [cited 2020 Sep 27]. Available from: https://pubmed. ncbi.nlm.nih.gov/30212513/.

13. PEPFAR. PEPFAR 2019 country operational plan guidance for all PEPFAR countries. 2019

14. Justman J. Presentation: recent infection testing in routine HIV testing services - overview from TRACE project; 2020.

15. Schlusser KE, Pilcher C, Kallas EG, Santos BR, Deeks SG, Facente S, et al. Comparison of cross-sectional HIV incidence assay results from dried blood spots and plasma. PLoS One. 2017;12(2) [cited 2020 Sep 27]. Available from: https://pubmed.ncbi.nlm.nih.gov/28231277/.

16. Eisenberg AL, Patel EU, Packman ZR, Fernandez RE, Piwowar-Manning E, Hamilton EL, et al. Short communication: dried blood spots stored at room temperature should not be used for hiv incidence testing. AIDS Res Hum 
Retrovir. 2018;34(12):1013-6 [cited 2020 Sep 27]. Available from: https:// pubmed.ncbi.nlm.nih.gov/30215267/.

17. Rice BD, de Wit M, Welty S, Risher K, Cowan FM, Murphy G, et al. Can HIV recent infection surveillance help us better understand where primary prevention efforts should be targeted? Results of three pilots integrating a recent infection testing algorithm into routine programme activities in Kenya and Zimbabwe. J Int AIDS Soc. 2020;23(S3):e25513. Available from: https://pubmed.ncbi.nlm.nih.gov/32602625/. [cited 2020 Sep 27]

18. Eastern Deanery AIDS Relief Program. Our background 2020. 2020. Available from: www.edarp.com

19. Welty S, Motoku J, Muriithi C, Rice B, de Wit M, Ashanda B, et al. Brief report: recent HIV infection surveillance in routine HIV testing in Nairobi, Kenya: a feasibility study. J Acquir Immune Defic Syndr. 2020;84(1):5. Available from: https://pubmed.ncbi.nlm.nih.gov/32058458/. [cited 2020 Sep 27]

20. Hargreaves JR, Mtetwa S, Davey C, Dirawo J, Chidiya S, Benedikt C, et al. Cohort analysis of program data to estimate HIV incidence and uptake of HIV-related services among female sex workers in Zimbabwe, 2009-2014. J Acquir Immune Defic Syndr. 2016;72(1):e1-8 [cited 2020 Sep 27]. Available from: https://pubmed.ncbi.nlm.nih.gov/27093516/.

21. Sedia Biosciences Corporation. Sedia HIV-1 LAg-Avidity EIA. Available from: http://www.sediabio.com/products/lag-avidity-eia. Accessed 28 Sept 2020.

22. Sedia Biosciences Corporation. Sedia HIV-1 LAg-Avidity EIA for Dried Blood Spots. Available from: http://www.sediabio.com/products/lag-avidity-eia-fordbs. Accessed 28 Sept 2020.

23. Maxim Biomedical Inc. Maxim HIV-1 Limiting Antigen Avidity (LAg-Avidity) EIA Kit, 192 tests. Available from: https://www.maximbio.com/Products/92 001/Maxim-HIV-1-Limiting-Antigen-Avidity-(LAg-Avidity)-EIA-Kit\%2C-192Tests. Accessed 28 Sept 2020.

24. Maxim Biomedical Inc. Maxim HIV-1 Limiting Antigen Avidity (LAg-Avidity) DBS EIA Kit, 192 tests. Available from: https:/www.maximbio.com/Products/ 92003/Maxim-HIV-1-Limiting-Antigen-Avidity-(LAg-Avidity)-DBS-EIA-Kit\%2C-1 92-Tests. Accessed 28 Sept 2020.

25. Maxim Biomedical Inc. Product Insert: Maxim HIV-1 Limiting Antigen Avidity EIA - Single Well Avidity Enzyme Immunoassay for Detection of Recent HIV1 Infection. Available from: https://www.maximbio.com/Products/92001/Ma xim-HIV-1-Limiting-Antigen-Avidity-(LAg-Avidity)-EIA-Kit\%2C-192-Tests. [Cited 2020 Sep 28]

26. Jung BH, Rezk NL, Bridges AS, Corbett AH, Kashuba ADM. Simultaneous determination of 17 antiretroviral drugs in human plasma for quantitative analysis with liquid chromatography-tandem mass spectrometry. Biomed Chromatogr. 2007;21(10):1095-104 [cited 2020 Sep 27]. Available from: https://pubmed.ncbi.nlm.nih.gov/17582235/.

27. Keating SM, Rountree W, Grebe E, Pappas AL, Stone M, Hampton D, et al. Development of an international external quality assurance program for HIV-1 incidence using the Limiting Antigen Avidity assay. PLoS One. 2019; 14(9) [cited 2020 Sep 27]. Available from: https://pubmed.ncbi.nlm.nih. gov/31525218/.

28. Stime KJ, Garrett N, Sookrajh Y, Dorward J, Dlamini N, Olowolagba A, et al. Clinic flow for STI, HIV, and TB patients in an urban infectious disease clinic offering point-of-care testing services in Durban, South Africa. BMC Health Serv Res. 2018;18(1):363 [cited 2020 Sep 27]. Available from: https://bmchea Ithservres.biomedcentral.com/articles/10.1186/s12913-018-3154-2.

\section{Publisher's Note}

Springer Nature remains neutral with regard to jurisdictional claims in published maps and institutional affiliations.

Ready to submit your research? Choose BMC and benefit from:
- fast, convenient online submission
- thorough peer review by experienced researchers in your field
- rapid publication on acceptance
- support for research data, including large and complex data types
- gold Open Access which fosters wider collaboration and increased citations
- maximum visibility for your research: over 100M website views per year
At BMC, research is always in progress.
Learn more biomedcentral.com/submissions

